\title{
NEUMONÍA POR BACTERIAS ATÍPICAS: MYCOPLASMA Y CHLAMYDIA PNEUMONIAE ¿QUÉ ELEMENTOS TENEMOS PARA HACER UN DIAGNÓSTICO ADECUADO Y DECIDIR CUÁNDO TRATAR?
}

Atypical pneumonia: mycoplasma and chlamydia pneumoniae. Making an accurate diagnosis and deciding when to treat

Dra Andrea Sepúlveda ${ }^{1}$, Dr Alex Castet ${ }^{1}$, Dr Pablo Bertrand ${ }^{2}$

1. Residente Pediatría. Pontificia Universidad Católica de Chile

2. Pediatra Especialista en Enfermedades Respiratorias, Profesor Asociado, Escuela de Medicina.

Pontificia Universidad Católica de Chile

\begin{abstract}
ATYPICAL PNEUMONIA: MYCOPLASMA AND CHLAMYDIA PNEUMONIAE. MAKING AN ACCURATE DIAGNOSIS AND DECIDING WHEN TO TREAT

Atypical Pneumonia has been studied for many years. Most clinically relevant atypical organisms involved in pneumonia in children are Mycoplasma pneumoniae and Chlamydia pneumoniae. Although great progress has been reached in new techniques, still there is no good tool, neither standardized nor accurate for a definitive diagnosis. In other hand, antibiotic therapy is under review due to contradictory evidence to support their use. We present a critical view of actual knowledge and propose an algorithm to proceed in clinical ground.
\end{abstract}

Key words: atypical pneumonia, Mycoplasma pneumoniae, Chlamydia pneumoniae

RESUMEN

La neumonía por bacterias atípicas es sujeto de estudio desde hace años. Dentro de las bacterias atípicas más frecuentes y clínicamente relevantes en niños se reconocen Mycoplasma pneumoniae y Chlamydia pneumoniae. A pesar del aumento en el conocimiento de estas infecciones y avance en las técnicas diagnósticas, aun no contamos con una herramienta estandarizada y confiable que permita realizar un adecuado diagnóstico. Por otra parte, la necesidad real de efectuar un tratamiento antibiótico sigue siendo tema de discusión. Se presenta a continuación una revisión crítica del conocimiento actual y una propuesta de su enfrentamiento clínico

Palabras clave: neumonía atípica, Mycoplasma pneumoniae, Chlamydia pneumoniae

\section{INTRODUCCIÓN}

La neumonía por bacterias atípicas, entendida como aquella que no sigue el curso clínico o radiológico habitual (1), ha sido tema de discusión desde su descripción. Se comenzó a mencionar como tal esta entidad cuando los conocimientos microbiológicos eran escasos y se limitaban a bacterias reconocibles con tinciones tradicionales como el Streptococo pneumoniae y Mycobacterium tuberculosis. Ya en 1938, en Philadelphia, Hobart Reimann popularizó el concepto a través de una publicación que reconocía algunas características especiales como el inicio gradual de la infección, poco compromiso general y asociación de síntomas respiratorios además de constitucionales, entre otras cosas. Algunos años más tarde, se estudió el comportamiento infeccioso de estas bacterias en la población de soldados estadounidenses de la Segunda Guerra Mundial, en los que se describen brotes de enfermedad (2). En la segunda mitad del siglo XX se fueron identificando distintos

Correspondencia:

Dr Pablo Bertrand

División Pediatría. P Universidad Católica de Chile

Lira 855 piso

E-mail; pbertrand@med.puc.cl agentes microbiológicos, que hoy en día constituyen el grupo de las Ilamadas bacterias atípicas. En este grupo destacamos Mycoplasma pneumoniae, Chlamydia pneumoniae, Chlamydia psitacci y Legionella pneumoniae.

La importancia actual de la infección por bacterias atípicas estriba en la alta frecuencia estimada, la ausencia de un método diagnóstico único idóneo y el conflicto de la necesidad real de tratamiento (3). Considerando estos elementos revisaremos la literatura al respecto centrando nuestra atención en Mycoplasma Pneumoniae y Chlamydia Pneumoniae.

\section{EPIDEMIOLOGÍA}

Es necesario aclarar que cuando nos referimos a neumonía atípica no lo hacemos en referencia a su frecuencia, sino todo lo contrario: las bacterias atípicas constituyen la segunda causa de neumonía en la población adolescente. Esto ha generado discrepancia en cuanto a seguir llamando este tipo de neumonía como "atípica". La literatura mundial señala que entre el 20 y $60 \%$ de los agentes infecciosos aislados en neumonías adquiridas en la comunidad correspondería a 
Mycoplasma Pneumoniae y/o Chlamydia Pneumoniae. En los reportes nacionales esta cifra alcanza entre el 17 y $34 \%$; sin embargo, estas cifras deber ser evaluadas con cautela, ya que son datos de poblaciones adultas y con métodos diagnósticos muy variables (2).

\section{MYCOPLASMA PNEUMONIAE}

El Mycoplasma pneumoniae es una bacteria muy pequeña, constituida por una triple membrana, carente de pared celular, razón por la cual no se tiñe al gram y es resistente a betalactámicos. Existen al menos 16 especies aisladas en humanos, de las cuales Mycoplasma pneumoniae es la más relevante y mejor conocida. Su pequeño genoma y capacidad biosintética limitada explica su crecimiento fastidioso y existencia parasitaria, con un período de incubación de 2 a 3 semanas. Dentro de sus componentes estructurales destaca la adhesina P1, proteína de membrana que es necesaria para su adherencia al epitelio respiratorio y que protege a la bacteria del clearance mucociliar. Luego de la adherencia la bacteria provoca ciliostasis, edema e infiltrado mononuclear y polimorfonuclear. Se ha descrito como potente mitógeno de linfocitos $T$ y B; esto último estaría relacionado con la producción de reacciones autoinmunes, dada la similitud de algunas adhesinas con HLA y algunos tejidos humanos (4).

La neumonía por Mycoplasma pneumoniae alcanza hasta un 40\% del total de las neumonías en niños. Tradicionalmente se ha postulado que se presenta preferentemente en escolares y adolescentes, sin embargo hoy se sabe que en preescolares la frecuencia es alta y probablemente sea esta población un reservorio para la infección a mayor edad $(4,5)$. Se presenta de forma endémica, con brotes en primavera y sobretodo en lugares o comunidades cerradas. Puede comprometer sólo la vía aérea superior en el 25\% de los casos, provocando una infección leve (otitis, faringitis) 0 asintomática (6). Los síntomas se desarrollan gradualmente en días a semanas. En lactantes la infección puede alcanzar hasta el 10\% del total de infecciones respiratorias del tracto inferior, y en escolares y adolescentes se encuentra con mayor frecuencia la progresión a neumonía. Dentro de los síntomas, la tos es el más constante descrito en la literatura, desde coqueluchoidea hasta productiva, en la mayoría de los casos de duración prolongada. Las manifestaciones pulmonares suelen ser similares a las producidas por infecciones virales como coriza, odinofagia y polipnea leve, destacando que además es muy frecuente la coinfección con virus (4).

En un estudio descriptivo realizado en el Hospital Clínico de la P. Universidad Católica de Chile entre los años 2000 y 2005, se analizaron las manifestaciones clínicas de pacientes cursando con neumonía por Mycoplasma pneumoniaey destaca que los síntomas referidos por los pacientes con mayor frecuencia fueron fiebre, compromiso del estado general y tos productiva. Con respecto a los signos, en la mitad de los pacientes se encontró sibilancias, y en alrededor de dos tercios de los casos crepitaciones (7). Una revisión Cochrane analiza los síntomas y signos clínicos de pacientes con infección por Mycoplasma pneumoniae para realizar el diagnostico de esta enfermedad. En esta publicación destaca que la ausencia de sibilancias está asociado a la presencia de la enfermedad y el dolor torácico dobla la probabilidad diagnóstica; sin embargo, la información obtenida en este estudio es insuficiente para establecer una relación entre la presencia/ausencia de estos y

\begin{tabular}{|c|c|}
\hline Tracto respiratorio superior & faringitis, otitis media, rinosinusitis \\
\hline Dermatológicas & rash eritematoso maculopapular, vesicular, eritema multiforme, síndrome de Steven Johnson \\
\hline Sistema nervioso central & $\begin{array}{c}\text { encefalitis, meningoencefalitis, meningitis aséptica, Guillain Barré, poliradiculitis, } \\
\text { mielitis transversa, diplopía, confusión }\end{array}$ \\
\hline Hematológicas & Anemia hemolítica \\
\hline Gastrointestinal & Náuseas, vómitos, diarrea \\
\hline Musculoesquelética & Mialgias, artralgias \\
\hline Cardiaca & Miocarditis \\
\hline Ocular & Conjuntivitis, uveítis, neuritis óptica, retinitis, hemorragia retinal, \\
\hline Renal & Glomerulonefritis \\
\hline & otros \\
\hline
\end{tabular}

Tabla 1. Manifestaciones extrapulmonares 
otros síntomas y el diagnóstico de la infección; Ilama la atención la ausencia de sibilancias, ya que por años se ha considerado un elemento altamente sugerente de esta etiología. Es necesario hacer el alcance de la necesidad de mejorar la evidencia, con estudios realizados en población ambulatoria y de mayor calidad metodológica. (8).

Las manifestaciones extrapulmonares ocurren entre el 25 y $50 \%$ de los casos y acompañan a las manifestaciones respiratorias en forma concomitante 0 diferida. (9) (Tabla 1). Las manifestaciones dermatológicas son las más frecuentes e incluyen desde rash eritematoso maculopapular, vesicular, hasta reacciones severas como eritema multiforme y síndrome de Steven Johnson.

Las complicaciones neurológicas son más frecuentes en pacientes de curso hospitalizado e incluyen entre otras: meningoencefalitis, meningitis aséptica, síndrome de Guillain Barré, polirradiculitis, mielitis transversa, síndrome de confusión, etc. Estas condiciones se presentan 1 a 2 semanas posterior a los síntomas respiratorios.
Otras manifestaciones extra respiratorias de la infección por Mycoplasma pneumoniae incluyen Anemia hemolítica, Artritis, Miocarditis, Pericarditis, Nefritis Intersticial, Uveitis y Conjuntivitis. Es altamente probable que los mecanismos patogénicos de las manifestaciones extra respiratorias estén en relación a reacciones de tipo inmune $(10,11)$.

En relación al compromiso radiológico, se ha descrito clásicamente un patrón reticular intersticial difuso, sin embargo muchos autores describen que los hallazgos en la radiografías de tórax pueden ser muy variados, y no permiten distinguirla de infección por bacterias típicas o agentes virales. Un elemento muy sugerente de infección por Mycoplasma pneumoniae es la clásica descripción de una disociación clínico-radiológica, en la que destacan muchos hallazgos radiológicos en un paciente poco sintomático o en muy buenas condiciones. De acuerdo al estudio realizado en el Hospital Clínico de la Universidad Católica, antes citado, lo más frecuente fue encontrar patrón de consolidación 0 infiltrado intersticial difuso (Figura 1)(7).

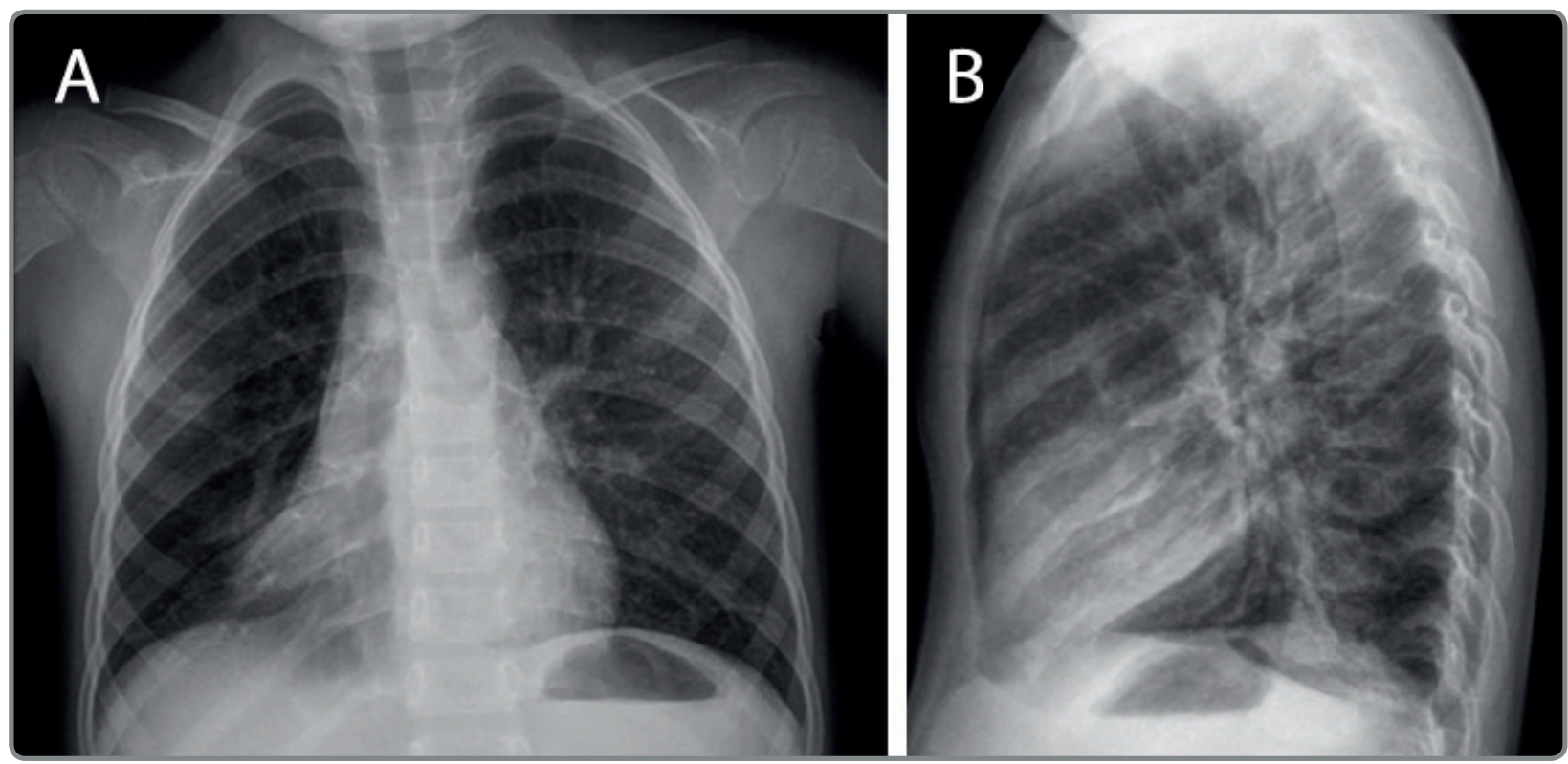

Figura 1. Radiografía de tórax anteroposterior (A) y lateral (B).

Paciente de 3 años y 4 meses. A. Radiografía anteroposterior: se observan sombras parenquimatosas en ambos lóbulos inferiores además de imagen condensante con pérdida de volumen del lóbulo medio que borra contorno cardíaco derecho. B. Lateral: sombras parenquimatosas que se proyectan en ambos lóbulos inferiores. Se confirma condensación con pérdida de volumen del lóbulo medio.

En relación al estudio de laboratorio específico del Mycoplasma pneumoniae, aún en la actualidad no existe una recomendación clara y única sobre la mejor prueba diagnóstica (Tabla 2). El cultivo es $100 \%$ específico para la presencia del agente pero no se utiliza porque requiere una incubación prolongada y la persistencia de la bacteria tras la infección puede durar semanas además que existe elevada portación que confunde con infección real $(12,13)$. 


\begin{tabular}{|l|c|c|}
\hline Prueba & Sensibilidad (\%) & Especificidad (\%) \\
\hline Cultivo & 61 & 100 \\
\hline Inmunofluorescencia indirecta & 78 & 92 \\
\hline ELISA & $93-100$ & $79-100$ \\
\hline Crioaglutininas & $30-50$ & 50 \\
\hline PCR & $78-100$ & $92-100$ \\
\hline
\end{tabular}

Tabla 2. Pruebas diagnósticas para Mycoplasma pneumoniae ELISA: enzyme-linked immunosorbent assay

PCR: polymerase chain reaction.

En cuanto a las técnicas serológicas por ELISA son de fácil realización, más sensible que cultivo para infección aguda y comparable a la reacción de polimerasa en cadena en inmunocompetentes. La IgM se eleva luego de la primera semana, y antecede en dos semanas la elevación de IgG; sin embargo, pueden detectarse IgM hasta 12 meses tras la infección aguda.

Las crioaglutininas son autoanticuerpos $\lg \mathrm{M}$, que generan aglutinación de eritrocitos a $4^{\circ} \mathrm{C}$ y se manifiestan en el $50 \%$ de las infecciones por Mycoplasma pneumoniae. Aparecen a las dos semanas y desaparecen a las 6 - 8 semanas. Actualmente están en desuso por tener baja sensibilidad y especificidad, ya que se producen en una variada gama de infecciones como Adenovirus y virus Epstein barr.

La reacción de polimerasa en cadena (PCR), es un método relativamente nuevo, que busca identificar regiones específicas del Mycoplasma pneumoniae (RNA ribosomal, adhesina, p1, entre otros). Esta técnica tiene las ventajas de que es altamente sensible, y rápida; sin embargo, al igual que el cultivo, la correlación clínica es fundamental para interpretar un resultado positivo como infección.

Realizar una recomendación en relación al estudio diagnóstico de Mycoplasma pneumoniae es complejo. Un estudio reciente ha comparado el uso de PCR versus serología. El hallazgo más importante mencionado en este estudio es la gran discordancia de resultados entre ambas pruebas, probablemente atribuible a la persistencia serológica de la bacteria. Los autores recomiendan el uso de ambas pruebas en forma complementaria (13). En relación a esto nos parece que una conducta prudente sería frente a una alta sospecha clínica de infección por
Mycoplasma, en el contexto de un paciente ambulatorio con enfermedad leve, considerarlo como infección, sin necesidad de realizar más test diagnósticos. Sin embargo en el caso de un paciente hospitalizado, con mayor compromiso, utilizar las técnicas disponibles, para lograr, junto a la evaluación clínica, una mejor interpretación de ellas.

En relación al tratamiento de la infección por Mycoplasma pneumoniae la droga de elección es un macrólido, la cual no tiene resistencia descrita y presenta la mayor capacidad de acumulación en el espacio intracelular (14). Entre estos, se prefiere azitromicina por su potencia y efecto prolongado, además que permite administración una vez al día, favoreciendo la adherencia. Sin embargo, el punto de discusión radica en la real necesidad y los objetivos del tratamiento farmacológico. Una revisión Cochrane que investigó la efectividad del tratamiento antibacteriano para infección por Mycoplasma pneumoniaeincluyó 7 ensayos clínicos randomizados, cuya mayor limitación es que los estudios incluían población infectada por otros agentes infecciosos. Se observó en esta revisión que no hubo diferencias en tratar a estos pacientes con macrólidos versus tratar con otro antibiótico inefectivo contra Mycoplasma pneumoniae (generalmente amoxicilina). Sólo un estudio mostró $100 \%$ resolución de síntomas en aquellos que recibieron azitromicina versus $77 \%$ en aquellos tratados con otros antibacterianos al mes de evolución. En esta revisión se concluye que la evidencia disponible es insuficiente para realizar una recomendación (15).

Una revisión más reciente con el mismo objetivo incluyó 17 estudios, muestra que la diferencia de riesgo que favorece el tratamiento no es significativa. Esta revisión menciona 
gran heterogeneidad entre los trabajos, con imposibilidad de corregir por test diagnóstico realizado, coinfección o tiempo de intervención, por lo que también concluye que la evidencia actual es insuficiente (16). Dado la evidencia mencionada, sigue siendo tema en discusión, quedando sujeto al criterio médico el hecho de tratar o no hacerlo. En relación a esto creemos que una conducta adecuada sería considerar la gravedad y compromiso clínico del paciente para tomar una conducta. Es decir, aquel paciente que cursa con neumonía que requiere hospitalización, que presenta evolución prolongada (> 14 días), o que se asocia a compromiso extrapulmonar debería recibir tratamiento antibiótico; por otro lado, un paciente que presenta síntomas leves, que no tiene enfermedad de base y cuya evolución clínica es benigna, podría recibir tratamiento sintomático bajo un seguimiento clínico cercano, dada la posibilidad de que se trate de un episodio auto limitado. De cualquier modo, ninguna recomendación reemplaza el criterio médico, que en cada situación debe considerar factores del paciente, del entorno epidemiológico y del tratamiento.

\section{CHLAMYDIA PNEUMONIAE}

Las Chlamydias son bacterias intracelulares, gram negativas, que contienen ADN y ARN ribosomal. Tienen un ciclo vital prolongado, de desarrollo en 2 fases: uno extracelular infeccioso y uno replicativo intracelular, que se comportan como repetidos ciclos de replicación binaria y diferenciación secundaria $(17,18)$. Codifican para una proteina estructural cuya síntesis es muy similar a la del peptidoglicano, incluyendo las proteínas de unión a penicilina (PBP). Esto explica la paradoja del peptidoglicano chlamydial, que la hace susceptible a betalactámicos.

La infección respiratoria por Chlamydia pneumoniae afecta a todas las edades y es de amplia distribución geográfica. Se trasmite y propaga por aerosol, sobretodo en núcleos cerrados. Las Chlamydias tienen la capacidad de sobrevivir en superficies por hasta 30 horas. Los reportes de incidencia son variables, asignándole la etiología de neumonía adquirida en la comunidad entre el 0 y $44 \%$ de los casos. Se ha descrito portación asintomática en el 2 a $5 \%$ de la población (4). Generalmente la infección por Chlamydia pneumoniae es poco sintomática y se confunde clínicamente con un resfrío común. Frecuentemente se presenta en coinfección con algún virus o Mycoplasma, quedando cubierta sus manifestaciones clínicas por las del coinfectante. En un porcentaje menor los niños infectados pueden presentar síntomas atribuibles a traqueobronquitis catarral y solo la minoría de los niños van a presentar síntomas por neumonía.

En relación a las pruebas diagnósticas no existe estandarización de una sola técnica. El cultivo se utiliza como estándar de oro, tomado de nasofaringe. En cuanto a la serología, la técnica más utilizada y de elección es el factor inhibitorio de macrófafos (MIF) que mide Inmunoglobulina (Ig)
M e IgG; la IgM aparece entre las 2 a 3 semanas, pero puede demorar hasta 6 semanas, la IgG aparece a las 8 semanas. Se ha establecido en forma estándar puntos de corte de $\lg \mathrm{M}>1: 16$ o IgG elevada sobre 4 veces su valor basal, sin embargo, existe gran heterogeneidad entre los laboratorios.

La PCR es una técnica cada vez más disponible; aun cuando los resultados son preliminares y la técnica no se ha estandarizado, parece ser que esta herramienta, de buena sensibilidad y especificidad, podría cambiar la práctica clínica por la rapidez en su disponibilidad (19)

En relación al tratamiento, a pesar de que existe susceptibilidad a betalactámicos, la mejor respuesta se logra usando agentes que actúan en la síntesis de proteínas como los macrólidos. De elección sería azitromicina, con lo que se erradica hasta el $80 \%$ del microorganismo (4). No existen estudios comparativos en relación a la efectividad del tratamiento antibiótico versus tratamiento sintomático; sin embargo, dado de que generalmente se trata episodios asintomáticos o levemente sintomáticos, la mayoría no llegará a plantear el dilema; por otra parte, si el cuadro clínico se manifiesta con síntomas de magnitud significativa, nuestra recomendación sería realizar el tratamiento ya sea bajo la sospecha de una infección o con diagnóstico confirmado por Chlamydia pneumoniae.

\section{CONCLUSIONES}

La infección por agentes atípicos sigue siendo tema de discusión en la actualidad. Si bien el término de "Neumonía atípica" ha perdido sentido al conocer la importancia en frecuencia de esta infección, la dificultad en el diagnóstico diferencial a través de la clínica, al menos en nuestro medio, lo hace todavía vigente. Está pendiente resolver cuál es la técnica de laboratorio más idónea para el diagnóstico y la real efectividad del tratamiento antibacteriano. En esta enfermedad, las habilidades médicas y el juicio clínico racional en la toma de decisiones son de mucho valor en virtud de los conocimientos existentes.

En la Figura 2 se propone un algoritmo para el enfrentamiento diagnóstico y terapéutico de la infección por estos agentes.

Los autores declaran no presentar conflicto de interés. 


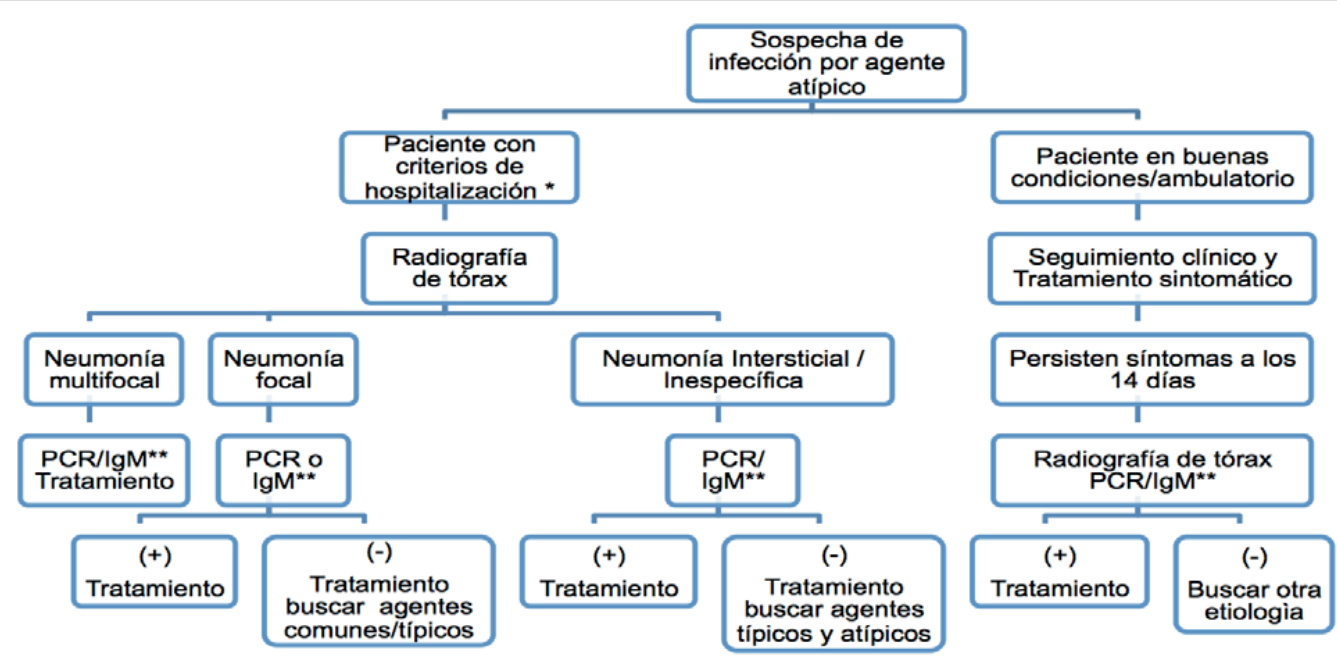

Figura 2. Algoritmo de manejo clínico en pacientes con infección por agentes atípicos

* Criterios de hospitalización: dificultad o insuficiencia respiratoria, requerimientos de oxígeno, compromiso extrapulmonar importante, sospecha de neumonía complicada o sepsis, neumonía, enfermedad subyacente

** Según disponibilidad

\section{REFERENCIAS}

1. Parra W. Neumonías atípicas. Neumol Pediatr 2013: 8:74-78

2. Murdoch D, Chambers S. Atypical pneumonia-time to breathe new life into a unuseful term? Lancet Infect Dis 2009; 9: 512-19 3. Wolf J, Daley A. Microbiological aspects of bacterial respiratory tract ilness in children: atypical patogens. Paediatr Respir Rev2007;8: 212-220

4. Waites K. New concepts of Mycoplasma infection in children. Pediatr Pulmonol 2003; 36:267-278

5. Baer G, Engelcke G, Abele-Horn M, Schaad U, Heininger U. Role of Chlamydia pneumoniae and Mycoplasma pneumoniae as causative agents of community acquired pneumonia in hospitalised children and adolescents. Eur J Clin Microbiol Infect Dis $2003 ; 22: 742-5$

6. Prinipi N, Esposito S. Emerging role of Mycoplasma pneumoniae and Chlamydia pneumoniae in paediatric respiratory tract infections. Lancet Infect Dis 2001; 1: 334-44

7.Paul M, Vega-Briceño L, Potin M, Ferrés M, Pulgar D, García C. Características clínicas de la enfermedad respiratoria causada por Mycoplasma pneumoniae en niños hospitalizados. Rev Chil Infect 2009; 26: 343-349

8. Wang K, Gill P, Perera R, Thomson A, Mant D, Harnden A. Clinical symptoms and signs for the diagnosis of Mycoplasma pneumoniae in children and adolescent with community acquired pneumoniae. Cochrane Database Syst Rev 2012 Issue 10.CD009175

9. Vervloet L, Marguet C, Moreira P. Infection of Mycoplasma pneumoniae and its importance as an etiological agent in childhood community acquired pneumoniae. BJID 2007;11:507514

10. Candler P, Dale R. Three cases of central nervous syndromes complications associated to Mycoplasma Pneumoniae. Pediatr Neurol 2004;31:133-138

11. Cherry J. Anemia and mucocutaneos lesions due to Mycoplasma pneumoniae infections. Clin Infect Dis 1993; 17 Suppl 1:S47

12. Loens K, Goossens H, leven M. Acute respiratory infection due to Mycoplasma pneumoniae: current status of diagnostic methods. Eur J Clin Microbiol Infect Dis 2010;29:1055-1069

13. Chang H, Chan L, Shao P, Lee P, Chen J, Lee C. Lu C. Huang $\mathrm{Li}$. Comparison of real-time polymerase chain reaction and serological test for the confirmation of Mycoplasma penumoniae infection in children with clinical diagnosis of atypical pneumonia. J Microbiol, Immunol Infect 2014; 47: 137-144

14. Allegra L, Blasi F. Problems and perspectives in the treatment of respiratory infections caused by atypical pathogens. Pulm Pharmacol Ther 2001; 14: 21-27

15. Mulholland S, Gavranich J, Gillies M, Chang A. Antibiotics for community acquired lower respiratory tract infections secondary to Mycoplasma pneumoniae in children. Cochrane Database Syst Rev. 2012 Issue 12;9:CD004875

16. Biondi E, McCulloh R, Alverson B, Klein A, Dixon A, Ralston S. Treatment of Mycoplasma penumoniae: A systematic review. Pediatr 2014;133:1081-1090

17. Hammerschlag M. Chlamydia trachomatis and Chlamydia pneumoniae infections in children and adolescents. Pediatr Rev 2004;25:43-51

18. Siqueira L. Chlamydia infection in children and adolescents. Pediatr Rev 2014;35;145-52

19. Kumar S, Hammerschlag M. Acute respiratory infections due to Chlamydia pneumoniae: current status of diagnostic methods. Clin Infect Dis 2007; 44:568-76 